\title{
Clinical and magnetic resonance imaging features of 'diamond on quadriceps' sign in dysferlinopathy
}

\author{
Sunil Pradhan \\ Department of Neurology Institute of Human Behaviour and Allied Sciences, Nerw Delhi and Sanjay Gandhi Postgraduate Institute of \\ Medical Sciences, Lucknow, India
}

Address for correspondence: Prof. Sunil Pradhan,

Department of Neurology Institute of Human Behaviour and Allied

Sciences, New Delhi - 110 095, India.

E-mail: drspradhan@ rediffmail.com

DOI: $10.4103 / 0028-3886.51287$

\begin{abstract}
Abstradt
There is very little by way of clinical examination that helps in the diagnosis of subtypes of limb-girdle muscular dystrophy (LGMD). A small observation led to this study on a clinical sign in a group of 31 patients with dysferlinopathy that included 13 with LGMD-2B and 18 with Miyoshi myopathy (MM). The patients were asked to stand with knees slightly bent so that the Quadriceps muscles were in moderate action. Those who could not stand in this posture due to muscle weakness were observed during sittingdown and standing-up in slow motion keeping hands away from thighs. Upper half of the anterolateral aspect of thighs was observed for any abnormal bulge. Twenty one (eight with LGMD-2B and 13 with MM) were found to have diamond-shaped bulge in this region with wasting of muscles above and below. This bulge was not apparent in standing or sitting position when Quadriceps muscle was not in action. T1-weighted MRI of the thighs revealed focal bulge-out of muscle fibers during contraction. Among the patients with other types of muscular dystrophy, who served as controls, one with clinical diagnosis of sarcoglycanopathy had multiple mild bulges visible over the Quadriceps muscle but none showed diamond-shaped or little elongated single bulge as was seen in dysferlinopathy. We infer that nearly two-thirds of the patients with dysferlinopathy demonstrate "diamond on Quadriceps' sign. Further studies are needed to look for its specificity among patients with muscular dystrophy.
\end{abstract}

Key words: C linical sign, dysferlinopathy, humans, imaging, muscular dystrophy

\section{Introduction}

Limb-girdle muscular dystrophy (LGMD) is a common term for several genetically different muscular dystrophies, which present often in adolescence or early adulthood with predominant weakness in girdle and proximal limb muscles. ${ }^{[1]}$ Clinical distinction is not possible due to lack of differentiating features and precise diagnosis depends upon the immunohistochemical study of known membrane proteins or the genetic testing. ${ }^{[1-3]}$ One of the sarcolemma-bound protein 'dysferlin', which is involved in membrane repair,,$^{[4]}$ is encoded by DYSF gene located at chromosome 2p13.[5] Two clinically distinct allelic forms are linked to DYSF gene - Miyoshi myopathy (MM) with wasting of distal limbs particularly leg muscles ${ }^{[6]}$ and
LGMD-2B with limb-girdle type of presentation in which the leg and other distal limb muscles remain preserved..$^{[7]}$ However, in view of common genetic defect ${ }^{[8]}$ and deficiency of gene product dysferlin, ${ }^{[4,9,10]}$ some common clinical features are expected to be present in these two disorders.

In the context of muscular dystrophy, it has been shown that certain muscles may exhibit enlargement of one part and wasting of another part. ${ }^{[11]}$ It has also been observed that subtle enlargement and wasting are better appreciated when the muscle is under mild contraction as happens when a particular posture is adopted. ${ }^{[11]}$ Based on these characteristics of muscular dystrophy, a new clinical signis described in dysferlinopathy. 


\section{M aterials and M ethods}

We studied 31 patients of dysferlinopathy out of whom 13 patients (from 11 families; 12 males and one female; aged 17-32 years with onset of illness between 15 and 28 years) suffered from LGMD-2B and 18 patients (from 12 families; 13 males and five females; aged $16-42$ years with onset of illness between 9 and 28 years) suffered from MM. The patients with LGMD-2B had primarily proximal muscle weakness that was more in the lower limbs than the upper. Those having MM type presentation had maximum weakness in the plantar-flexors and dorsi-flexors of the feet with varying degree of weakness in proximal lower limb muscles, knee flexors being slightly more wasted than the knee extensors. In the upper limbs, MM patients showed mild wasting of biceps, triceps, and forearm muscles. Rest of the muscles at shoulders and upper back showed the same pattern of wasting, enlargement, and contractures as described earlier in 'calf-head sign', ${ }^{[12]}$ the latter being also convincingly observable in 10 patients with MM.

The diagnosis of dysferlinopathy was based on: (1) typical pattern of gradually progressive wasting and weakness of muscles that conformed to either LGMD2B or MM, (2) moderately raised creatine kinase levels, (3) myopathic pattern on electromyography, and (4) the absence of dysferlin on immunostaining of muscle biopsy specimens.

\section{Elicitation of the sign}

All patients with dysferlinopathy were asked to stand with knees slightly flexed so that the quadriceps femoris muscles were in action. Those who could not sustain this posture for long were asked to gradually sit down to ground (or to a chair) and to gradually get up in slow motion. Patients were also asked to keep both hands wide apart so that their anterolateral aspect of thighs could be fully visualized. The sign was considered positive when a diamond-shaped bulge stood out at the anterolateral aspect of the middle of thighs that disappeared in normal standing or sitting position when the quadriceps muscle was not in action.

Patients with other muscular dystrophies and spinal muscular atrophies were also examined in this manner to look for the sign. These included 15 patients with Becker muscular dystrophy, 16 patients with facioscapulohumeral dystrophy, 14 with myotonic dystrophy, 15 with sarcoglycanopathy, 5 with congenital myopathy, and 11 with spinal muscular atrophy type III.

\section{Magnetic resonance imaging}

MRI of the thighs was performed on two patients with MM and two with LGMD-2B who demonstrated the clinical sign. It was done on a 1.5-Tesla superconducting
MRI machine (Lx Echo speed plus, GE Medical System, Milwaukee, USA) using body coil. T1-weighted coronal oblique imaging was done using spin echo sequence with the following parameters: $\mathrm{TR}=500 \mathrm{msec}, \mathrm{TE}=$ $15 \mathrm{msec}, \mathrm{Nex}=4, \mathrm{FOV}=340 \mathrm{~mm}$. The slice thickness was $5 \mathrm{~mm}$ with no interslice gap and $36 \times 512$ matrix. The patient lay supine with knees slightly flexed and feet resting on a footrest. Longitudinal sections were obtained in the plane at variable angles from the coronal plane depending upon the direction of the clinically visible bulge. After obtaining MR images in the resting state, patients were asked to press the footrest distally to obtain steady contraction of quadriceps muscle; MRI was obtained again in this state. Axial sections were also obtained both in relaxed and contracted state of quadriceps muscle in the region that showed muscle bulge in oblique coronal plane.

\section{Results}

'Diamond on quadriceps' sign was present in 21 out of 31 (68\%) patients with dysferlinopathy that included 8 out of $13(62 \%)$ with LGMD-2B and 13 of the 18 (71\%) with MM. One patient with MM was unable to get up or stay standing with bent knees; he was asked to voluntarily contract his quadriceps muscle and this act was enough to elicit the sign. One patient with MM needed partial support to get up from sitting position and this provided enough contraction of quadriceps muscle to produce the expected sign. The 'diamond on quadriceps' sign was observable in a subtle manner in six LGMD-2B and five MM patients, while it was overtly present in two LGMD-2B see [Figure 1] and eight MM patients. Out of the 10 patients with absent sign, two (one from each group) had advanced stage of the disease with generalized muscle wasting and two patients of LGMD-2B were in a very early stage of the disease with only slight difficulty in getting up for one and

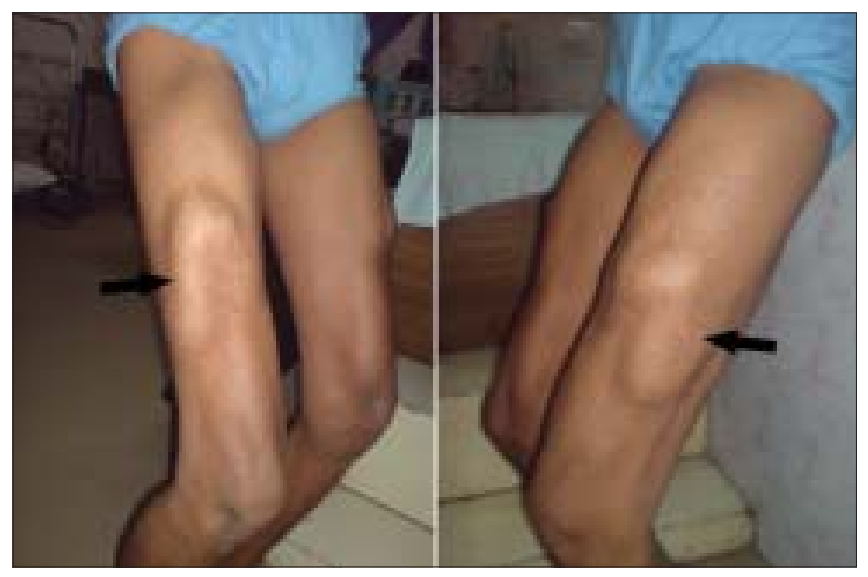

Figure 1: 'Diamond on quadriceps' sign in a case of LGMD-2B. Note the diamond-shaped solitary lumps on anterolateral aspect of the quadriceps muscle on both sides (arrows) and the standard posture adopted by patients to look for the sign 
three years. All the patients with positive sign had moderate disability of more than three years of illness. The diamond-shaped lump that bulged out during contraction of the quadriceps femoris muscle was always a single lump on each quadriceps muscle in front of the thigh in its rectus femoris component in two patients and on anterolateral or lateral aspect [Figure 1] of thigh in its vastus lateralis component in rest of them.

T1-weighted MRI of the quadriceps femoris muscle showed mild generalized wasting of thigh muscles that was apparently more in the posterior aspect. The quadriceps femoris muscle had uniform texture and smooth surface at rest [Figure 2a]. When made to contract, a portion of the muscle bulged out toward the anterolateral aspect [Figure 2b] near the middle of the thigh [Figure 2c].

\section{Discussion}

Enlargement of some muscles and wasting of some other individual or group of muscles is well known in muscular dystrophies..$^{[1-3]}$ What is less well known, however, is the fact that different parts of a particular single muscle may also show enlargement in one part and wasting in another part. ${ }^{[1]]}$ For example, in Duchenne muscular dystrophy it has been demonstrated that the muscle fibers of vastus lateralis part of the quadriceps muscle are often enlarged but that of vastus medialis part are wasted in their whole length. Sometimes a segment along the length of the muscle is enlarged and another segment is wasted. For example, enlarged fibers of deltoid and infraspinatus muscles in Duchenne muscular dystrophy show wasting in parts that traverse and contribute to the posterior axillary fold..$^{[11-13]}$ Similarly in facioscapulohumeral dystrophy deltoid muscle fibers are well preserved or enlarged in lower two-thirds of their length but are wasted in upper one-third. ${ }^{[14]}$ The selective wasting, selective preserved bulk, and the selective enlargement of whole or part of the muscle that becomes more conspicuous when the muscles are in action, as happens when a patient adopts a particular posture, has been made the basis of several of the clinical signs described recently. ${ }^{[12-17]}$ In this context, the present study demonstrates that dysferlinopathies have a tendency for wasting of quadriceps femoris muscle, particularly its two components vastus lateralis and rectus femoris, in their upper and lower parts with some sparing or somewhat enlargement in the middle part. The demarcation between wasted and the spared segments of the muscle fibers is so sharp that whenever the muscle is in action its central part bulges out in the shape of a diamond. ${ }^{[18]}$

MRI features have been described previously in MM. With regard to distal wasting it has been found that the imaging abnormalities may precede the clinical symptoms. ${ }^{[1,20]}$ All the four of our patients whose MRI was done, were in the symptomatic stage of the disease and it was not possible to observe whether MRI features precede the clinical observation. As the fibers in quadriceps femoris muscle are obliquely placed our MRI findings suggest that the dystrophic process in dysferlinopathies start at both the terminals of this muscle and that the fibers in the mid part remains intact till late during the process of degeneration. This observation however, requires confirmation from autopsy studies.

We observed multiple lumps upon contraction of quadriceps muscle in one patient with sarcoglycanopathy. Such 'lumps and bumps' have been described previously by Khadilkar et al., in patients with sarcoglycanopathies. ${ }^{[21]}$ This implies that the presence of lumps over the quadriceps muscle will help in narrowing down the differential diagnosis of muscular dystrophy and in this

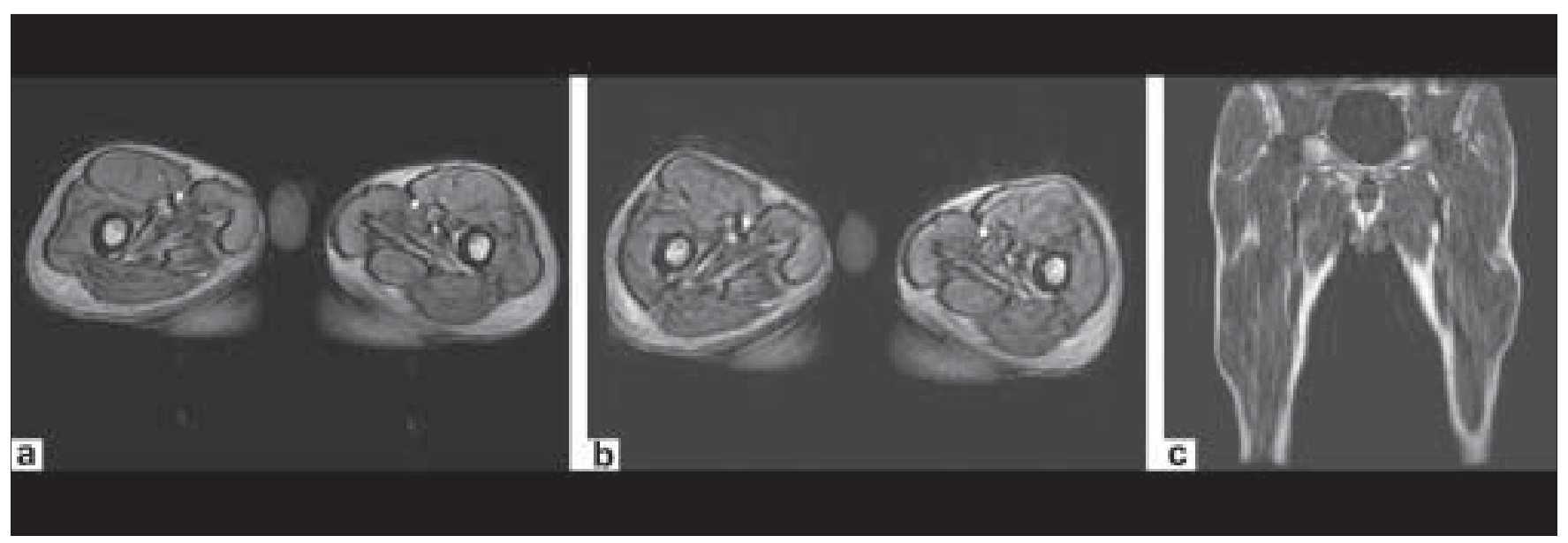

Figure 2: T1-weighted MRI showing axial section in relaxed state (a) axial section in contracted state (b) and oblique coronal section in contracted state (c) of quadriceps femoris muscle in a patient of Miyoshi myopathy. Note the muscle bulge toward the anterolateral aspect (b) near the middle of the thigh (c) in contracted state. Also, note the similarity between the clinical [Figure 1] and MRI features 
context it needs further clarification whether the single rather than multiple lumps seen in our patients with dysferlinopathy has any specificity or not. Further, it appears that dysferlinopathy has a tendency for lump formation in some other muscles as well. Biceps lump has been described previously by Fardeu and colleagues from Paris and by Khadilkar and Singh from India. ${ }^{[22]}$ However, biceps lump has also been reported in an anecdotal case of facioscapulohumeral dystrophy. ${ }^{[23]}$ These observations suggest that the lump formation in muscles could be highly suggestive of dysferlinopathy, but one must take into consideration the other muscular dystrophies where lumps have been described and take help of other clinical features to make a clinical diagnosis.

The 'diamond on quadriceps' sign appears to be a common sign in patients with dysferlinopathy, seen nearly in two-thirds of the patients with LGMD-2B as well as MM subtypes. However, its specificity and sensitivity needs to be evaluated taking larger number of patients into consideration. As the diagnosis of dysferlinopathy nowadays is made by demonstrating absence of dysferlin in muscle membrane on immunohistochemical staining of muscle biopsy specimens or by genetic study, this sign would help in suspecting patients on clinical grounds so that they could be directed to appropriate tests, thus minimizing the time and cost of final diagnosis.

\section{References}

1. Amato AA, Brooke MH. Disorders of skeletal muscle. In: Bradley WG, Daroff RB, Fenichel GM, Jankovic J, editors. Neurology in clinical practice - the neurological disorders [Vol. II]. $4^{\text {th }}$ Ed. Boston: Butterworth - Heinemann 2004. p. 2463-510.

2. Morgan-Hughes JA. Diseases of striated muscles. In: Asbury AK, McKhann GM, McDonald WI, eds. Diseases of the nervous system - clinical neurobiology, Vol. 1. Philadelphia: W. B. Saunders Company 1992. p. 164-96.

3. Riggs JE, Schochet SS. Muscle disease. In: Joynt RJ, editor. Clinical neurology [Vol. 4, chap. 53]. Philadelphia: J. B. Lippencott Company 1994. p. 1-35.

4. Bansal D, Miyake K, Vogel SS, Groh S, Chen CC, Williamson R, et al. Defective membrane repair in dysferlin-deficient muscular dystrophy. Nature 2003;423:168-72.

5. Bashir R, Strachan T, Keers S, Stephenson A, Mahjneh I, Marconi G, et al. A gene for autosomal recessive limb-girdle muscular dystrophy maps to chromosome 2p. Hum Mol Genet 1994;3:455-7.

6. Bejaoui K, Hirabayashi K, Hentati F, Haines JL, Ben Hamida C, Belal S, et al. Linkage of Miyoshi myopathy (distal autosomal muscular dystrophy) locus to chromosome 2p12-14. Neurology 1995;45:768-72.

7. Bashir R, Keers S, Strachan T, Passos-Bueno R, Zatz M, Weissenbach J, et al. Genetic and physical mapping at the limb-girdle muscular dystrophy locus (LGMD 2B) on chromosome 2p. Genomics 1996;33: 46-52.

8. Liu J, Aoki M, Illa I, Wu C, Fardeau M, Angelini C, et al. Dysferlin, a novel skeletal muscle gene, is mutated in Miyoshi myopathy and limb girdle muscular dystrophy. Nat Gen 1998;20:31-6.

9. Matsuda C, Aoki M, Hayashi YK, Ho MF, Arahata K, Brown RH. Dysferlin is a surface membrane-associated protein that is absent in Miyoshi myopathy. Neurology 1999;53:1119-2.

10. Anderson LVB, Davison K, Moss JA, Young C, Cullen MJ, Walsh J, et al. Dysferlin is a plasma membrane protein and is expressed early in human development. Hum Mol Genet 1999;8:855-61.

11. Pradhan S, Mittal B. Infraspinatus muscle hypertrophy and axillary folds wasting as the important signs in Duchenne muscular dystrophy. Clin Neurol Neurosurg 1995;91:134-8.

12. Pradhan S. Calf-Head sign in Miyoshi myopathy. Arch Neurol 2006;63:1414-7.

13. Pradhan S. New clinical sign in Duchenne muscular dystrophy. Pediatr Neurol 1994;11:298-300.

14. Pradhan S. Poly-hill sign in facioscapulohumeral dystrophy. Muscle Nerve 2002;25:754-5.

15. Pradhan S. Shank sign in myotonic dystrophy type-I (DM-I): Utility in differentiation from DM-II and some other common muscular dystrophies. J Clin Neurosci 2007;14:27-32.

16. Pradhan S. Valley sign in Duchenne muscular dystrophy: Importance in patients with inconspicuous calves. Neurol India 2002;50:184-6.

17. Pradhan S. Valley sign in Becker muscular dystrophy and DMD/BMD outliers. Neurol India 2004;52:203-5.

18. Pradhan S. Diamonds on quadriceps sign in dysferlinopathy. Neurology 2008; $70: 322$.

19. Meola G, Sansone V, Rotondo G, Jabour A. A computerized tomography and magnetic resonance muscle imaging in Miyoshi's myopathy. Muscle Nerve 1996;19:1476-80.

20. Cupler EJ, Bohlega S, Hessler R, McLean D, Stigsby B, Ahamad J. Miyoshi myopathy in Saudi Arabia: Clinical, electrophysiological, histopathological and radiological features. Neuromusc Disord 1998;8:321-6.

21. Khadilkar SV, Singh RK, Katrak SM. Sareoglyeanopathies: A report of 25 cases. Neurol India 2002;50:27-32.

22. Khadilkar SV, Singh RK. Limb girdle muscular dystrophy in India. Neurol India 2008;56:281-8.

23. Pradhan S. An extra hill on a poly-hill sign in a patient with facioscapulohumeral dystrophy. Neurol India 2007;55:436-7.

Accepted on 31-03-2009

Source of Support: Nil, Conflict of Interest: None declared. 